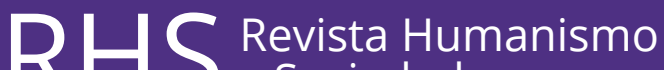 \\ has
}

\section{Un acercamiento a los enfoques de investigación y tradiciones investigativas en educación}

\author{
An approach to research viewpoints and traditions in education \\ Nelson D'olivares Durán ${ }^{1^{*}}$, Clara Liliana Casteblanco Cifuentes ${ }^{2 * *}$ \\ ${ }^{1}$ linguisticamaestra@gmail.com; 2 lailamaestra@gmail.com
}

Este artículo es una revisión teórica acerca de los enfoques de investigación. Hace parte de del grupo de investigación Filosofía, Sociedad y Educación, UPTC.

Recibido: octubre 13 de 2015. Aceptado: noviembre 24 de 2015.

doi: 10.22209/rhs.v3n1.2a04

\section{Resumen}

Este artículo ofrece orientaciones y material de consulta para que sirva de referencia a los investigadores interesados tanto en enfoques como en tradiciones investigativas, y puedan llevar a cabo estudios en educación. Por ello, introduce un esbozo teórico de los enfoques cuantitativo, cualitativo y mixto de investigación. Además, relaciona algunas tradiciones investigativas entre las que se encuentran la investigación acción, la investigación acción participativa, el estudio de caso y la investigación etnometodológica.

Palabras clave: investigación cuantitativa, investigación cualitativa, tradiciones investigativas.

\begin{abstract}
This paper provides guidance and reference material to researchers interested in both research approaches and research traditions to carry out investigations in education. We present a theoretical framework about quantitative, qualitative and mixed research approaches. In addition, this document reviews some research traditions such as action research, participatory action research, case study, and ethnomethodological research.
\end{abstract}

Keywords: quantitative research, qualitative research, research traditions.

Para citar este artículo: D'olivares Durán, N. \& Casteblanco Cifuentes, C.L. (2015). Un acercamiento a los enfoques de investigación y tradiciones investigativas en educación. Rev Humanismo y Sociedad, 3(1-2), 24-34. doi: 10.22209/rhs.v3n1.2a04

* Seminarista Misionero-mxy; Licenciado en Idiomas Modernos Español-Inglés - Universidad Pedagógica y Tecnológica de Colombia (UPTC); candidato a Magíster en Lingüística - UPTC; doctorando en Lenguaje y Cultura - UPTC. Investigador del grupo de investigación Filosofía, Sociedad y Educación - UPTC. Profesor tiempo completo Universidad de Cundinamarca.

** Licenciada en Educación Preescolar - UPTC; aspirante a Magíster en Educación - UPTC; adscrita al grupo de investigación filosofía, sociedad y educación de la Maestría en Educación - UPTC; investigadora de la línea de Filosofía e Infancia - UPTC. Profesora catedrática - UPTC. Intercesora de la Fundación Pedagógica Rayuela, Tunja. 


\section{Introducción}

En este documento nos ocuparemos brevemente de los enfoques de investigación en educación y algunas tradiciones investigativas. Se llega a la concepción de este escrito, merced de un ejercicio de reflexión y constante diálogo con colegas y doctores al seno de uno de los seminarios en torno de los métodos de investigación en educación del Doctorado en Lenguaje y Cultura de la Universidad Pedagógica y Tecnológica de Colombia, y que como profesores investigadores demanda lectura, comprensión y análisis de la bibliografía que este programa y que esta clase de seminarios precisa, ya sea esta de carácter obligatoria, complementaria o particular. Con todo, estamos en la búsqueda y producción de conocimiento centrados en la investigación académica y científica.

En este artículo, primero se plantea una noción de investigación como el proceso de búsqueda de modos posibles para la solución de un problema mediante acciones como describir, comprender, analizar y explicar. Luego, se abordan los enfoques de investigación y sus características propias: cuantitativo, cualitativo y mixto, con los cuales a través de sus procesos de observación y evaluación de fenómenos, formulación de hipótesis, pruebas y análisis, se plantean otras observaciones y evaluaciones para explicar, variar y cimentar las hipótesis o para concebir otras. Enseguida, se enlistan algunas tradiciones investigativas tales como la investigación acción, la investigación acción participativa, el estudio de caso, entre otras. Finalmente, una síntesis cierra el artículo.

\section{Un acercamiento a los enfoques de investigación}

La ciencia se manifiesta de modo claro a través de la investigación. La ciencia es un conocimiento producido por el ser humano con el objetivo de explicar el porqué de las cosas. Así, la investigación se ocupa de las maneras y estrategias utilizadas por los investigadores para entender el mundo que nos rodea. En el proceso de investigación se observa y se describe para entender y evaluar lo que está sucediendo, por más simple que sea el suceso, la investigación exige rigurosas pruebas para que una verdad sea aceptada. Justamente, la investigación es una suma de procesos sistemáticos, críticos y empíricos que se aplican al estudio de un fenómeno.
En otras palabras, la investigación es el proceso mediante el cual un sujeto se interesa en lograr conocimiento o nuevo conocimiento de un hecho o fenómeno. Pongo por caso, el proceso de búsqueda de modos posibles para la solución de un problema: describir, comprender, analizar y explicar para llegar a una posible solución o una posible respuesta. En este sentido, replicar un método o construirlo, ya sea cuantitativo en tanto recolección de datos, etc., o cualitativo en tanto sentido, comprensión, entendimiento, etc., o mixto, llevará a relacionar criterios en el campo o campos de interés del investigador y a contribuir con el mejoramiento del conocimiento.

\section{Enfoques en la investigación}

La Historia de la ciencia nos ha puesto a disposición diversas corrientes de pensamiento para orientar el conocimiento: empirismo, materialismo dialéctico, positivismo, fenomenología, estructuralismo, y otras interpretativas como la etnografía y el constructivismo. Pero, dadas las diferentes premisas propuestas por tales corrientes, desde el siglo xx se han concentrado en dos enfoques importantes para investigar: el enfoque cuantitativo y el enfoque cualitativo. Estos dos enfoques utilizan procesos sesudos, sistemáticos y experimentales en su quehacer para construir conocimiento.

De acuerdo con Grinnell (1997) en términos generales, tanto el enfoque cuantitativo como el cualitativo aplican cinco pasos muy similares: observación y evaluación de fenómenos; surgen hipótesis como resultado de la observación y evaluación; demuestran en qué medida las hipótesis tienen soporte; estudian tales hipótesis a través de la prueba o el análisis; plantean nuevas observaciones y evaluaciones para explicar, variar y cimentar las hipótesis o para concebir otras.

Ahora bien, los dos enfoques siguen esos pasos generales, pero cada enfoque tiene características propias.

\section{Características del enfoque cuantitativo}

El enfoque cuantitativo es una suma de procesos secuenciales y probatorios. Todos los pasos van escalonados, es decir que uno antecede al siguiente, permite rediseñar algún paso, pero no omitirlo. Tiene un orden juicioso. Inicia con una idea que se delimita, para luego, formular objetivos y preguntas de investigación, 
en seguida se revisa la literatura y se construye un marco o una perspectiva teórica.

A partir de las preguntas surgen las hipótesis y se establecen variables; se planea un diseño para probar las hipótesis; se miden las variables en un contexto dado; se estudian las mediciones logradas y se analizan a través de técnicas estadísticas, y se establecen conclusiones en relación con las hipótesis.

En este enfoque el investigador hace planteamientos de problemas de estudio delimitados sobre asuntos concretos. Luego, el investigador hace la revisión de la literatura para seguirle la pista a lo que se ha investigado antes al respecto y poder construir un marco teórico que guiará su trabajo, y de allí resultarán las hipótesis que va a estudiar si son ciertas o no, enseguida las pone a prueba con un diseño de investigación ajustado a su trabajo.

Si las consecuencias de esas pruebas confirman las hipótesis, se toma como evidencia para confiar en la teoría que las respalda. Si contradicen las hipótesis, se anulan en procura de encontrar otras explicaciones y se establecen nuevas hipótesis y, si se quiere, nuevas teorías que las apoyen. Enseguida, se pasa a la recolección de los datos fundamentados en la medición de las variables percibidas en las hipótesis. Se recurre a maneras de recolección de datos ajustados y reconocidos por una comunidad científica, demostrando que se siguieron dichas maneras o procedimientos y así la investigación será aceptada por otros investigadores.

Como el enfoque cuantitativo pretende medir, se trabaja con fenómenos que se observan o están referidos en la realidad, por tal, los datos resultan de mediciones que se cuantifican y se analizan mediante técnicas estadísticas. En la recolección de datos, este enfoque busca el máximo control para prescindir de la incertidumbre, disminuir el error y descartar posibles explicaciones distintas de la hipótesis, por esto, se confía en la experimentación y los ensayos de causa-efecto.

Para Creswell (2005) después de la recolección de datos, se pasa al análisis de los datos que son interpretados teniendo en cuenta las hipótesis y las teorías de estudios previos, así, la interpretación de los datos da cuenta de cómo los resultados se ensamblan en el conocimiento existente.
Según Unrau, Grinnell y Williams (2005) conviene subrayar que la investigación cuantitativa apunta a ser lo más «objetiva» posible para no ser alterada, por lo cual el investigador evita que sus puntos de vista o sesgos, o los de otros, tengan alguna injerencia en los resultados de la investigación o en su proceso, es decir que la investigación cuantitativa acaece en circunstancias externas al investigador. De esta manera, las investigaciones cuantitativas sufren un proceso previsible y ordenado, donde las decisiones críticas tienen lugar previo de recolectar los datos; y de acuerdo con ciertas reglas lógicas, los datos obtenidos tienen los estándares de validez y confiabilidad, y las conclusiones resultantes aportarán a la generación de conocimiento.

Es de notar que la meta primordial de los estudios cuantitativos es la construcción y demostración de teorías para explicar y predecir los fenómenos estudiados, buscando puntos en común y aspectos emergentes entre elementos. Por esto, en un estudio cuantitativo se procura generalizar los resultados arrojados por una muestra a una población o universo.

Bergman (2008) afirma que la investigación cuantitativa pretende identificar leyes universales y causales, valiéndose de la lógica (razonamiento deductivo), que principia con la teoría, de la cual se salen las hipótesis (expresiones lógicas), que a su vez, el investigador pone a prueba.

El enfoque cuantitativo es más comúnmente aplicado a investigaciones en el campo de las ciencias naturales, esto depende de la pregunta-variable objeto de estudio.

\section{¿Cómo se concibe la realidad en el enfoque cuantitativo?}

Traemos a cuento las teorías de Grinnell (1997) y Creswell (1997) quienes plantean, por un lado, una realidad interna que son las creencias, presupuestos, intuiciones y experiencias personales, las cuales varían desde nimiedades o globales hasta opiniones establecidas lógicamente mediante teorías formales. Por otro lado, una segunda realidad que es objetiva, autónoma y externa al investigador, es decir, una realidad que acaece sin importar lo que pensemos o nuestras creencias sobre ella; por ejemplo, la religión, el herpes, una casa, etc. Así, esta segunda realidad está 
dispuesta para ser conocida. De este modo, es viable investigar la realidad objetiva.

Para ello, es necesario tener un alto nivel de comprensión de la realidad objetiva, y para empezar a comprenderla hay que registrar los fenómenos y los eventos que la rodean, por decir, que un metal que se supone tiene una propiedad dada legítimamente la posea, la relación entre los motivos de una persona y su conducta, que las causas que inducen una enfermedad sean verdaderas, etc. No hay que apresurarse a pensar que en el enfoque cuantitativo lo subjetivo no exista o no tenga un cierto valor para los investigadores, cosa distinta es que el enfoque cuantitativo se centra en demostrar cuán bien encaja el conocimiento en la realidad objetiva.

\section{Enfoque cualitativo}

Al igual que el enfoque cuantitativo, el cualitativo también se deja guiar por asuntos específicos de investigación. Sin embargo, la investigación cualitativa puede plantear preguntas e hipótesis antes, durante o después de la recolección y el análisis de los datos.

Este procedimiento, pese a su complejidad y flexibilidad, es provechoso, ya que permite enunciar preguntas auxiliares de investigación, pulirlas para formular la más significativa. En este enfoque, la actividad investigativa oscila entre lo que acaece y su interpretación. Así, el enfoque cualitativo apunta más a un proceso circular que no sigue necesariamente un canon establecido, es decir, que la sucesión de los pasos se altera según el estudio.

Es necesario decir que este es un intento por abordar el enfoque cualitativo de investigación dada su complejidad, y que es preciso anotar las siguientes observaciones.

En el enfoque cualitativo hay una revisión inicial de la literatura, pero esta no es definitiva sino que se complementa en cualquier paso de la investigación, y que las teorías apoyan desde el planteamiento del problema de investigación hasta la producción de las conclusiones. Este enfoque permite que la investigación regrese a etapas previas o ajustes si es necesario. Respecto del investigador, este se sumerge en el campo de estudio para encontrarle significado al entorno, seleccionar informantes, confrontar la viabilidad del estudio, entre otros. En relación con la muestra, y el proceso de recolección y análisis de datos, se puede decir que se lleva a cabo simultáneamente.

\section{Características del enfoque cualitativo}

Este enfoque toma el contexto natural del hecho o fenómeno y al investigador como su componente vital de dicho contexto, quien va al punto de partida o fuente de datos y se interesa por el proceso y por los resultados. De acuerdo con Esterberg (2002) la investigación cualitativa inicia explorando, examinando y describiendo lo que ocurre en el mundo social, y en este proceso genera perspectivas teóricas afines con los datos. Pongo por caso, en un proyecto con enfoque cualitativo, el investigador lleva a cabo unas entrevistas, las analiza para sacar unas conclusiones; luego, realiza otras entrevistas con otros informantes, las analiza y revisa las conclusiones; y sigue haciendo el mismo proceso hasta llegar a comprender lo que busca. En otras palabras, el investigador procede entrevista por entrevista hasta alcanzar una vista más general.

Ahora es oportuno decir que, normalmente, en el enfoque cualitativo no se prueban hipótesis, sino que a lo largo del proceso se van concibiendo y puliendo a medida que se recogen los datos, o se conciben como un resultado de la investigación. Para la recolección de datos se utilizan métodos como la observación, revisión de documentos, discusiones en grupo, registro de historias de vida, blog, portafolio, diario de campo, entrevista semidirigida, etc., ya que la recolección de datos tiene que ver con puntos de vista, experiencias, significados, emociones, prioridades, interacciones entre personas o comunidades, y demás aspectos subjetivos de los participantes.

En concordancia con Todd, Nerlich y McKeown (2004) el investigador se interesa por vivencias y asuntos abiertos, recoge datos tanto a través del código escrito, verbal y no verbal, como visual, les hace descripción y análisis para relacionarlos con su tema; por tal razón, la medición no es numérica ni estadística. En algunos casos, la investigación está determinada por el contexto, luego los resultados no buscan establecer generalidades y las conclusiones desembocan en la población objeto de estudio.

De lo anterior se infiere que el proceso investigativo es más flexible y oscila entre las respuestas y el tratamiento de la teoría. Así, el propósito del enfoque cualitativo reside en reconstruir la realidad sin manipularla, para 
hacer una interpretación de ella y entender el significado de la realidad de los seres humanos." Justamente, para este enfoque, la realidad se delimita a partir de las interpretaciones de los participantes en relación con sus propias realidades. Se comprende, pues, que en este proceso confluyen la realidad de los participantes, la del investigador y la que produce la interacción de todos los actores; y que dichas realidades se van modificando a medida que avanza el estudio y se toman como fuentes de datos.

De ello resulta que el investigador se sumerge en las experiencias de los participantes para construir el conocimiento, él es consciente de que hace parte del fenómeno estudiado, salvo algunas excepciones en que el investigador podría no ser parte del fenómeno y podría no ser consciente de cuándo es o no parte del fenómeno estudiado. Por consiguiente, el centro del estudio cualitativo es la pluralidad de ideologías y cualidades únicas de las personas. No parece excesivo afirmar que el enfoque cualitativo se puede concebir como un acervo de prácticas interpretativas que le permiten al mundo ser visible, y que tales prácticas interpretativas transforman y convierten a dicho mundo en una sucesión de representaciones a manera de observaciones, registro de grabaciones, apuntes y documentos.

El enfoque cualitativo es aplicado con más frecuencia a investigaciones trabajadas en el campo de las ciencias sociales, esto obedece al tipo de pregunta-variable objeto de estudio.

\section{Enfoque mixto}

De acuerdo con Chen (2006), Johnson y Onwuegbuzie (2004) el enfoque mixto de investigación es la combinación sistemática del enfoque cuantitativo y el enfoque cualitativo en el mismo estudio para obtener un panorama más completo del fenómeno que se está estudiando. Se puede abordar el enfoque mixto manteniendo los procedimientos propios de cada enfoque, o

\footnotetext{
* La investigación cualitativa busca interpretar las acciones de los seres vivos, sobre todo de los seres humanos y sus instituciones; en ese sentido, es naturalista porque estudia a los objetos y seres vivos en sus contextos o ambientes naturales y cotidianidad, e interpretativo pues intenta encontrar sentido a los fenómenos en función de los significados que las personas les otorguen.
}

el investigador puede ajustarlos, adecuarlos, alterarlos o adaptarlos para llevar a cabo su estudio.

Es de aclarar que tanto el enfoque cuantitativo como el cualitativo son igual de importantes y fructíferos, ninguno es mejor que el otro, ambos le han aportado a la ciencia; uno y otro componen su enfoque respecto del estudio de un fenómeno determinado. Asimismo, el enfoque mixto de investigación comporta la misma importancia del cuantitativo y el cualitativo, pues, muchas preguntas de las ciencias naturales serán resueltas únicamente y de forma adecuada por un enfoque cuantitativo, similarmente, en las ciencias sociales y el enfoque cualitativo.

\section{Razón de ser del enfoque mixto}

Para Hernández Sampieri y Mendoza (2008), Creswell, Plano-Clark y Garret (2008) hoy por hoy, las ciencias enfrentan fenómenos complicados y diversos, como el consumo de drogas, la interacción de un individuo o un grupo, el suicidio, el SIDA, la crisis de los paperos, la ludopatía, el hambre, entre muchos más, que el uso únicamente del enfoque cuantitativo o cualitativo no da abasto para abarcar estos problemas; es por esto que aparece el enfoque mixto.

Creswell (2009), Newman, Ridenour y de Marco (2002) afirman que en la actualidad la investigación debe ser un trabajo multidisciplinario, que incluya investigadores con modos de investigar diversos, así se abarca el fenómeno más integral, amplia y profundamente.

La investigación mixta se apropia de las fortalezas de los métodos cualitativo y cuantitativo para indagar las diferentes aristas surgidas, de repente, en algún estadio del proceso investigativo. Creswell (2005) expone que el enfoque mixto contiene mayor riqueza de abordaje del problema. Miles y Huberman (1994) lo designan como «mayor poder de entendimiento». Harré y Crystal (2004) señalan que esta constitución entre lo cualitativo y lo cuantitativo nutre el poder de medición del fenómeno. Lincoln y Guba (2000) anotan que tanto el enfoque cuantitativo como el cualitativo suministran una «fotografía» de la realidad. 
Para Onwuegbuzie y Leech (2005) las similitudes entre el enfoque cuantitativo y cualitativo abundan; además, sea cual sea la epistemología,

all research in the behavioral and social sciences represent an attempt to understand human behavior (Onwuegbuzie and Leech, 2005). Thus, it is clear that if differences prevail between quantitative and qualitative researchers, these discrepancies do not arise from different goals. Instead they occur because the two groups of investigators have operationalized their strategies differently for reaching these goals (Dzurec and Abraham, 1993). This suggests that methodological pluralism should be enhanced. The best way to accomplish this is for as many investigators as possible to become pragmatist researchers.

A través del enfoque mixto, el investigador debe confrontar las resistencias de las nociones teóricas, a la vez que sopesa la relación entre los datos recogidos por los diferentes enfoques; En palabras de Todd, Nerlich y McKeown (2004) el enfoque mixto produce más riqueza en los datos dada la diversidad de observaciones desde los diferentes entornos, contextos y análisis, es decir, se rompe con la investigación uniforme y se llevan a cabo investigaciones más dinámicas, con una muestra más enriquecida, con mayor fidelidad del instrumento, con integridad en la intervención. Siguiendo a Creswell et al. (2008) en el enfoque mixto se saca beneficio de datos cuantitativos y cualitativos dentro del mismo estudio.

Algunos procesos investigativos han sido desarrollados en el marco de un enfoque cuantitativo o cualitativo y han tenido que acudir al otro enfoque para complementar la investigación. Además, Onwuegbuzie y Leech (2005) sugieren:

By having a positive attitude towards both techniques, pragmatist researchers are in a better position to use qualitative research to inform the quantitative portion of research studies, and vice versa. In particular, pragmatist researchers also are more able to combine empirical precision with descriptive precision. Armed with a bifocal lens, rather than with a single lens, pragmatist researchers will be able to zoom in to microscopic detail or to zoom out to indefinite scope (Willems and Raush, 1969).

Se puede sacar en limpio que los enfoques cuantitativo y cualitativo pueden cohabitar sin designar mayor o menor importancia a alguno de los dos. Así, varios autores como Onwuegbuzie y Leech (2005) y Brannen (2008) afirman que por décadas hemos utilizado y aprendido de ambos enfoques, los cuales han tenido injerencia en el quehacer académico.

\section{Algunas tradiciones investigativas}

El investigador logra abordar la realidad de su objeto de estudio mediante la observación y medición (datos cuantitativos) o la conceptualización y contextualización (datos cualitativos) y, de esta manera, llegar a describirlo, comprenderlo, analizarlo y explicarlo. Ahora bien, si el interés del investigador se centra en el proceso es recomendable usar el enfoque cualitativo, pero si el interés del investigador se centra en el resultado es recomendable usar el enfoque cuantitativo. Las siguientes tradiciones investigativas provienen de la investigación según el enfoque analítico del objeto de estudio, exactamente de la investigación cualitativa.

El tratamiento que aquí se le da a las distintas tradiciones investigativas más comunes es somero, consideramos que en aras de disipar dudas al respecto, es menester dedicar un capítulo completo enfocado exclusivamente a ellas. Además, se debe decir que en este artículo, tradición investigativa se refiere al modo de abordar un proceso investigativo dentro del enfoque cualitativo de investigación, otros autores usan términos como marcos interpretativos, diseños, tipologías, entre otros.

\section{Investigación Acción}

Esta tradición investigativa (IA) se lleva a cabo como experiencia por uno o varios investigadores y se deriva de un grupo social; el proceso investigativo puede iniciar con un solo individuo para, más adelante, ser fundamentado en el grupo social, y así lograr una reflexión crítica frente al plan de acción, dado que los productos serán empleados en dicho grupo social; pongo por caso:

Ejemplo 1. El uso de una estrategia para mejorar el proceso de enseñanza-aprendizaje del idioma inglés.

En este caso, el investigador (profesor) procura una o varias opciones de trabajo y adquiere un compromiso con ellas, mientras que los estudiantes se apropian de esas alternativas. Es así como el método adquiere una importancia inherente a esta tradición investigativa, de acuerdo con Firestone (1987), citado por D'Olivares (2013, p. 64), concibe la investigación acción «as a systematic process of inquiry which aims at looking 
for answers to questions or solutions to problems in a consistent, objective way by gathering, analyzing and interpreting information». De esta manera, esta tradición señala un modo en espiral para recolectar información, partiendo de un plan, una acción, una observación y una reflexión.

La tradición de investigación acción es muy habitual en el contexto educativo, por ello, Kemmis y McTaggart (2000), citado por D'Olivares (2013, p. 64), afirman que «action research is used to refer to teacher-initiated classroom investigation which seeks to increase the teacher's understanding of classroom practices». Notemos, además, que el método en esta tradición investigativa sigue unas etapas que pueden estar diseñadas de la siguiente forma para el ejemplo 1:

- Hacer una revisión del objeto de estudio para identificar un problema.

- Planeación y diseño de estrategias para mejorar el proceso de enseñanza-aprendizaje.

- Práctica, aplicación o acción de estrategias designadas.

- Estudio reflexivo de lo observado.

- Evaluación hecha por parte de los involucrados.

- Convalidación y publicación de los resultados.

\section{Investigación Acción Participativa}

En esta tradición investigativa se analizan las soluciones en acuerdo con el grupo. Es decir, todos los miembros están involucrados, tanto el investigador como el grupo social investigado, o lo que es lo mismo, el investigador los hace partícipes de la solución de problemas, innovación o búsqueda de medios para satisfacer las necesidades de dicho grupo. La metodología de esta tradición investigativa está basada en la práctica al servicio del ser humano, y se relaciona íntimamente con los hombres y las mujeres. Las etapas de esta tradición investigativa (IAP) pueden ser: exploración del grupo social, descripción de la problemática, elaboración y aplicación o acción de estrategias. Pueden estar planteadas de la siguiente forma:

- Selección de un grupo social dado.

- Definir necesidades del grupo social.

- Definir facilidades para realizar el trabajo

- Revisión de datos.

- Formar equipos de trabajo
- Especificar el objetivo y el proceso.

- Establecer funciones y cronograma.

- Definir estructura administrativa (grupos de trabajo, equipo coordinador y líder).

- Desarrollo del trabajo.

- Información continua a todos los miembros del grupo acerca.de adelantos (la observación participante compromete a los miembros como informante de su propio problema).

- Retroalimentación y búsqueda de soluciones (romper barreras entre investigador y objeto de estudio o personas estudiadas).

- Socialización de resultados al grupo social.

- Discusión y análisis.

- Búsqueda de estrategias de mejoramiento.

De acuerdo con Murcia (1992) esta tradición investigativa (IAP) hace menos hincapié en el conocimiento científico y le da prelación al conocimiento práctico que aparece en el grupo social que está siendo estudiado.

\section{Estudio de caso}

En esta tradición investigativa se construye conocimiento mediante la indagación de problemas del entorno propios del día a día del grupo social estudiado. A propósito del campo educativo, Wassermann (2006, p. 20) afirma que un caso es «el vehículo mediante el cual se lleva al salón de clase un trozo de realidad a fin de que los estudiantes y el profesor lo examinen minuciosamente». Es decir, se toma por caso la descripción de un hecho, en la vida de alguien o de un grupo, que para su estudio tiene en cuenta la realidad en todas sus proporciones. Esta tradición investigativa no es exclusiva de una única forma de ser interpretada, asimismo sugiere diferentes soluciones.

En muchas oportunidades, los casos abordan asuntos concretos tales como educación (conductas de los estudiantes, grado de lectura, preferencias profesionales, etc.), problemas sociales (alcoholismo, drogadicción, delincuencia juvenil, etc.), política (corrupción, conflictos internacionales, etc.), entre otros; sin embargo, los casos per se son interdisciplinarios. En pocas palabras, los casos pueden ser abordados para su estudio desde diversos campos, ya que el caso no aporta respuestas sino que expone datos específicos listos para ser analizados con rigor, para que los involucrados en el proceso encuentren aquello a lo que le deben dar solución. Asimismo, la tradición investigativa estudio de caso incluye las siguientes fases: establecer la línea inicial, 
introducir el tratamiento y hacer el análisis del cambio. Podemos proceder de la siguiente forma:

- Recoger datos

- Preparar situaciones interesantes

- Concebir categorías de estudio

- Seleccionar datos que suministren insumos para las categorías de estudio

- Buscar variedad en las categorías de estudio

- Explorar, describir y justificar las categorías del interés del investigador

- Trabajar con los datos emergentes

- Codificar y analizar las categorías principales

La tradición investigativa estudio de caso se puede esquematizar así: 0 corresponde a las continuas observaciones, y X a la variable independiente (o tratamiento).

$000 \times 000$

\section{Tradición investigativa etnometodológica}

El papel que desempeña esta tradición se basa en estudiar el modo como los sujetos implementan las normas culturales ideales y la aprehensión de sentido común a escenarios específicos, para desambiguar las acciones, ya que para los investigadores de esta tradición, los sentidos de las acciones son ambiguos, confusos y complejos. Es así como los etnometodólogos comprueban y explican las diversas estrategias que usan los sujetos para darle sentido a sus ocupaciones cotidianas.

De estas circunstancias nace el hecho de que la investigación etnometodológica* estudia la idea de que el mundo social comprende significados y puntos de vista compartidos, es decir, los significados asignados a las cosas son una obtención de los miembros de un grupo social. Esta tradición investigativa muestra que las acciones de los sujetos se pueden explicar solo dentro del contexto del cual sucedieron, según Garfinkel (en Hill y Crittenden 1968, p. 208), citado por Firth (2010, p. 604), «puede hablar de un tipo de reflexividad interminable que tienen los relatos, de forma que la "conversación" redunda en el escenario en el cual ocurre para ilustrar las características de tal escenario y, de ese modo, ilustrar también las características propias de la conversación». Además, la investigación

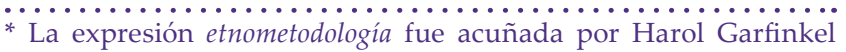
para referirse a las prácticas discursivas de los sujetos en contextos sociales. etnometodológica estudia el análisis conversacional a partir del diálogo natural y espontáneo, la coherencia y el cambio de turnos.

\section{Etnografía}

Esta tradición investigativa se sustenta en que la certeza de los valores, roles, tradiciones y reglas del entorno en que se vive son interiorizados paulatinamente por los miembros de una comunidad, lo cual origina una suerte de observancias que sirven para dar explicación conveniente a comportamientos y procederes individuales y grupales, ya que los miembros de un grupo comparten estructuras que generalmente no están manifiestas, pero que se presentan en diferentes facetas de la vida.

Para Martínez (1991, p. 16) el «objetivo inmediato de un estudio etnográfico es crear una imagen realista y fiel del grupo estudiado, pero su intención y mira más lejana es contribuir en la comprensión de sectores o grupos poblacionales más amplios que tienen características similares». Lo cual quiere decir que se trata de comprender aquello que acontece al interior de una comunidad y, para lograr este objetivo, además de habitar con dicha comunidad, hay que valerse de una revisión minuciosa del estado del arte y así establecer comparaciones o relaciones entre las investigaciones existentes.

Por consiguiente, la unidad de análisis de la etnografía $^{* *}$ es el ethnos, traducido en una comunidad lingüística, una región, o un grupo social con identidad y que esté regulado por la costumbre, derechos y obligaciones mutuos entre sus miembros. Podemos decir que esta tradición investigativa se subdivide en descriptiva cuando tiene que ver con el lenguaje verbal y no verbal; y aplicada cuando tiene que ver directamente con la antropología y la sociología.

De acuerdo con Castillo et al. (2001), la etnografía se desarrolla en tres etapas: descriptiva, interpretativa y construcción teórica.

\section{Descriptiva:}

- Formulación de preguntas

- Definición de instrumentos para la recopilación

** La palabra etnografía proviene de la antropología. y según Wo...... (1987, p. 18) la palabra etnografía significa la descripción (grafé) del estilo de vida de un grupo de seres humanos habituadas a vivir juntas (ethnos). 
de la información (trabajo de campo: diarios, entrevistas, audiovisuales, observación participante, historias de vida, cuestionarios, cuaderno de apuntes, etc.)

- Establecimiento de categorías

- Formulación de hipótesis

Interpretativa:

- Comprobación o comparación de hipótesis para reestructurar las propuestas o planteamientos.

Construcción teórica:

- Planteamiento de argumentos provenientes de la observación y los datos conseguidos, relacionando el conocimiento social diario con el conocimiento científico.

Esto nos lleva a decir que la tradición etnográfica registra una realidad vivida por el etnógrafo y donde no se descuida la red de relaciones que componen la realidad de una comunidad.

\section{Fenomenología}

Esta tradición investigativa se centra en la experiencia (fenómeno) particular de cada miembro del grupo involucrado en el proceso investigativo. La fenomenología se apoya en cuatro pilares: tiempo, espacio (lugar), corporalidad (persona física) y el contexto relacional (o comunidad), así, esta tradición pone el acento en la comprensión del significado de lo vivido (fenómeno) por cada individuo, pues es a través de cómo vive el mundo que el individuo demuestra su existencia. Precisamente, una mejor comprensión de la estructura de la experiencia vivida (fenómeno) permite reconocer la existencia de un significado. Para Merleau-Ponty (1976, p. 209) «las estructuras no pueden ser definidas como cosas del mundo físico, sino como conjuntos percibidos y, esencialmente, consisten en una red de relaciones percibidas, que, más que conocida, es vivida», bien se comprende que la estructura no se explica a partir de la realidad exterior, sino a partir del conocimiento, teniendo en cuenta que son percepciones y no realidades materiales o tangibles.

De acuerdo con Álvarez-Gayou (2009) esta tradición investigativa se basa en proposiciones tales como:

- Se describe para entender las experiencias vividas o fenómenos desde la percepción individual y colectiva.
- Se analizan discursos para encontrar sus significados.

- A través de su intuición y saberes interiorizados, el fenomenólogo comprende las experiencias de los partícipes en el proceso investigativo.

- El fenomenólogo asienta las experiencias o fenómenos a partir del tiempo y el espacio o lugar en que ocurrió lo vivido, de la corporalidad o las personas físicas que vivieron la experiencia y el contexto relacional o los vínculos originados en las experiencias con la comunidad.

- Los datos recogidos a través de los instrumentos, entrevistas, historias de vida, etc., servirán para hallar más temas acerca de fenómenos tanto habituales como inusuales.

- Los datos recogidos arrojan información sobre los involucrados en el proceso investigativo y sobre el fenómeno investigado.

Acudimos a este ejemplo para ilustrar un poco más acerca de la fenomenología. Colombia es un país azotado por el cruel flagelo del secuestro, a partir de allí, se puede hacer un estudio con la candidata presidencial y su jefa de debate liberadas en la «operación jaque», el hijo del padre que caminó más de 1000 kilómetros, el diputado del Valle del Cauca, el gobernador del Meta, los ingenieros de petróleos estadounidenses, entre otros, para investigar y comprender cómo ellos definen y describen esa terrorífica experiencia vivida o fenómeno desde la comprensión individual y colectiva.

La fenomenología, al igual que las anteriores y las demás tradiciones que comporta el enfoque cualitativo, se identifican porque la experiencia de los sujetos partícipes del proceso investigativo son la médula de la investigación.

En aras de la brevedad, y para no alargarnos con otras tradiciones investigativas solo vamos a dejar enunciadas las siguientes: comunidad de indagación, paradigma indiciario, narrativa, grupos focales, teoría fundamentada, entre otras.

\section{Conclusiones}

Sintetizando, diremos para terminar que la investigación es la clara manifestación de la ciencia, la cual es un conocimiento producido a través de procesos sistemáticos y empíricos por investigadores, con el objetivo de explicar el porqué de las cosas. La investigación es 
aplicada para ambos enfoques: cuantitativo y cualitativo. En primer lugar, el cuantitativo es una suma de procesos escalonados y probatorios, para permitir el rediseño de alguno de dichos procesos y no prescindir de él. Este enfoque tiene un orden estructurado, inicia con una idea que se delimita, se formulan objetivos y preguntas, se construye un marco teórico, surgen hipótesis y se establecen variables, se prueban las hipótesis, se miden las variables, se estudian y se analizan las mediciones logradas a través de técnicas estadísticas, y se establecen conclusiones.

En segundo lugar, en el enfoque cualitativo se realiza una revisión inicial de la literatura que se va complementando sobre la marcha desde el planteamiento del problema hasta las conclusiones. Este enfoque permite regresar y hacer ajustes en etapas previas. El investigador hace inmersión en el campo de estudio, se seleccionan informantes, se verifica la viabilidad del estudio, entre otros. La muestra, recolección y análisis de datos se puede realizar simultáneamente.

Por su parte, el enfoque mixto demanda un trabajo multidisciplinario con investigadores diversos para abarcar el fenómeno estudiado más integral, amplia y profundamente. La investigación mixta se vale de los enfoques cualitativo y cuantitativo para suministrar una fotografía de la realidad, apropiándose de sus fortalezas para aumentar la riqueza de abordaje del problema en alguna etapa del proceso investigativo y, de este modo, nutrir el poder de medición de los fenómenos.

Lo anterior se relaciona también con la tradición investigativa, la cual hace referencia al modo de abordar un proceso investigativo dentro del enfoque cualitativo de investigación. Dentro de esas tradiciones investigativas encontramos algunas, a saber, investigación acción, investigación acción participativa, estudio de caso, etnometodológica, etnografía y fenomenología.

En este artículo nos dedicamos a revisar y aclarar los diferentes enfoques de investigación y algunas tradiciones investigativas a partir del enfoque cualitativo, con la firme intención de que las ideas discutidas en estas líneas produzcan nuevas ideas que contribuyan a otros investigadores, de algún modo, con asertos epistemológicos para estudios futuros que aborden temas relacionados con investigación en educación e investigación en lenguaje, sociedad y cultura.

\section{Referencias}

Álvarez-Gayou J.L. (2009). Cómo hacer investigación cualitativa. Fundamentos y metodología. México: Paidós.

Bergam, M.M. (2008). Introduction: Whiter mixed methods? En M.M. Bergam (ed.), Advances in mixed methods research (p. 17). Thousand Oaks, CA, EE. UU: Sage.

Brannen, J. (2008). The practice of mixed methods research strategy: Personal, professional and project considerations. En M.M. Bergam (ed.), Advances in mixed methods research (pp. 53-65). Thousand Oaks, CA, EE. UU.: Sage Publications Inc.

Castillo, N., Jaimes, G. \& Chaparro R. (2001). Una aproximación a la investigación cualitativa. Tunja: UPTC.

Chen, H.T. (2006). A Theory-driven Evaluation Perspective on Mixed Methods Research. Research in the schools, 13(1), 75-83.

Creswell, J.W. (2005). Educational research: Planing, conducting, and evaluating quantitative and qualitative research (2a Ed.). Upper saddle River, NJ, EE. UU. Prentice Hall.

Creswell, J.W., Plano Clark, V. L. \& Garrett, A.L. (2008). Methodological issues in conducting mixed methods research designs. En M.M. Bergam (ed.), Advances in mixed methods research (pp. 66-83). Thousand Oaks, CA, EE. UU.: Sage.

Creswell, J. W. (2009). Research design: Qualitative, quantitative and mixed approaches (3a Ed.). Thousand Oaks, CA, EE. UU.: Sage.

D'Olivares, N. (2013). Cooperative learning: another way to learn together. Enletawa (6), 59-72. Tunja: UPTC.

Esterberg, K. G. (2002). Qualitative methods in social research. New York, NY, EE. UU.: McGraw-Hill.

Firth, A. (2010). Etnometodología. Discurso y Sociedad, 4(3), 597-614. Traducido por Teresa E. Cadavid G. Newcastle University, United Kingdom. www.dissoc.org 
Grinnell, R. M. (1997). Social work research and evaluation: Quantitative and qualitative approaches (5a Ed.). I Tasca, IL: F.E. Peacock.

Harré, R. \& Cristal, D. (2004). Discursive analisis and the interpretation of statistics. En Z. Todd, B. Nerlich, S. McKeown \& D. Clarke (Eds.), Mixing methods in psychology (pp. 61-80). Brighton/Hove, Sussex, UK: Psychology Press.

Hernández, S. \& Mendoza C.P. (2008). El matrimonio cuantitativo-cualitativo: el paradigma mixto. En J. L. Álvarez Gayou (Presidente), 6 Congreso de Investigación en Sexología. Congreso efectuado por el Instituto Mexicano de Sexología, A. C. y la Universidad Juárez Autónoma de Tabasco, Villahermosa, Tabasco, México.

Johnson, R. B. \& Onwuegbuzie, A. J. (2004). Mixed methods research: A research paradigm whose time has come. Educational Researcher, 33(7), 14-26.

Lincoln, Y. S. \& Guba, E. G. (2000). Paradigmatic controversies, contradictions, and emerging confluences. En N. K. Denzine Y. S. Lincoln (Eds.) Handbook of qualitative research (2 ${ }^{\mathrm{a}} \mathrm{Ed}$.) (pp. 163-188). Thousand Oaks, CA, EE. UU.: Sage.

Martínez, M. (1991). La Investigación Cualitativa Etnográfica en Educación. (6 $6^{\mathrm{a}}$ Ed.). Círculo de lectura Alternativa. Bogotá.

Merleau-Ponty, M. (1976). Estructura del comportamiento. Buenos Aires, Argentina: Hachette.

Miles, M. B. \& Huberman, A.M. (1994). Typology of sampling strategies qualitative inquiry: A source of new methods. Thousand Oaks, CA, EE. UU.: Sage.
Murcia-Florián, J. (1983). Elementos para la investigación en la Facultad de Psicología. Bogotá, Colombia: Magisterio.

Newman, I., Ridenour, C. S., Newman, C. \& De Marco, G. M. (2002). A typology of research purposes and its relationship to mixed methods in social and behavioral research (pp. 167-188). Thousand Oaks, CA, EE. UU.: Sage.

Onwuegbuzie A. J. \& Leech. (2005). Taking the "Q" Out of Research: Teaching Research Methodology Courses Without the Divide Between Quantitative and Qualitative Paradigms. Quality \& Quantity, 39(3), 267-295.

Onwuegbuzie, A.J., Slate, J.R., Leech, N. \& Collins, K. M. (2010). Mixed research: A step-by-step guide. London, UK: Taylor and Francis.

Todd, Z., Nerlich, B. \& McKeown, S. (2004). Introduction. En Z. Todd, B. Nerlich, S. McKeown \& D. Clarke (Eds.), Mixing methods in psychology (pp. 3-16). Brighton/Hove, Sussex, UK: Psychology Press.

Unrau, Y. A., Grinell, R. M. \& Williams, M. (2005). The quantitative research approach. En $M$. R. Grinnell e Y. A. Unrau (Eds.), Social work: Research and evaluation. Quantitative and qualitative approaches (7a Ed.) (pp. 61-73). New York, NY, EE. UU.: Oxford University Press.

Wassermann, S. (2006). El estudio de casos como método de enseñanza. Buenos Aires, Argentina: Amorrortu.

Woods, P. (1987). La escuela por dentro. La etnografía en la investigación educativa. Barcelona, España: Paidós. 